\title{
Retinal Changes in Severe Malaria in Bangladeshi Children
}

\author{
ABUL KALAM AZAD ${ }^{1}$, SANAT KUMAR BARUA ${ }^{2}$, FARID UDDIN AHMED ${ }^{3}$, PARVEZ IQBAL SHARIF ${ }^{4}$, \\ NASIR UDDIN MAHMUD 5
}

\begin{abstract}
:
Background: In malaria endemic areas diagnosis of severe malaria by microscopy and immunodiagnostic test is confounded by asymptomatic peripheral blood parasitemia. In such settings, retinal changes by ophthalmoscopy showed some diagnostic utility. Contribution of ophthalmoscopy in diagnosis of severe malaria in children is not well studied in Bangladesh. The aim of this study was to describe the retinal findings in children of cerebral and non-cerebral severe malaria by direct and indirect ophthalmoscope and relate their outcome and to determine the course of changes in the fundus.

Methodology: In this prospective observational study 130 consecutive children aged between 6 months to 12 years admitted with confirmed severe Plasmodium falciparum malaria in pediatrics ward of Chittagong Medical College Hospital, Chittagong, Bangladesh were assessed by both direct and indirect ophthalmoscopy during the period of April 2008 to March 2009.

Results: Out of 130 patients 80 children had cerebral malaria and of these 49 (61.2\%) had some degree of retinopathy; 24 (48\%) of 50 with non cerebral severe malaria had retinopathy. Predominant retinal changes were Retinal hemorrhage and Macular whitening each in $53(40.77 \%)$ patients, followed by peripheral whitening, Vessels changes and papilloedema in 50 (38.46\%), 40 (30.77\%) and 14 (10.78\%) patients respectively. With indirect ophthalmoscope as reference, direct ophthalmoscopy had a high sensitivity to detect macular whitening, and papilloedema but was less sensitive to detect peripheral whitening and vessel changes. Patients with retinopathy had more chance to had unfavorable outcome (Death) in comparison to the patients who had not \{Odds ratio:1.09 (95\% Cl:1.017-1.167)\}. Most of these retinal changes were transient and resolved gradually as clinical condition improved.

Conclusion:Features compatible with malarial retinopathy were commonly found in our children with severe malaria. Ophthalmoscopy is an important clinical tool to aid in diagnosis and prognosis in children. However, indirect ophthalmoscopy provides better information than direct ophthalmoscopy.
\end{abstract}

Key words: Cerebral malaria, Severe malaria, Retinopathy, Indirect ophthalmoscopy, Bangladeshi child.

1. Consultant, Paediatrics:Central Point Hospital, Sawndip, Chattogram, Bangladesh.

2. Associate Professor, Department of Paediatrics Nephrology, Chittagong Medical College Hospital, Chattogram, Bangladesh.

3. Emergency Medical Officer, Chittagong Medical College and Hospital, Chattogram, Bangladesh.

4. Associate Professor, Community Medicine, Chattogram International Medical College, Chattogram, Bangladesh.

5. Professor, Department of Paediatrics, Chittagong Medical College Hospital, Chattogram, Bangladesh.

Correspondence to: Prof. Dr. Nasir Uddin Mahmud, Professor, Department of Paediatrics, Chittagong Medical College Hospital, Chittagong, Bangladesh, Mobile: 01819379833, Email: nasirmahmudcmc24@gmail.com

Received: 26 September 2018 Accepted: 25 October 2018

\section{Introduction:}

More than 1 million people die of severe and cerebral malaria (CM) every year in the world. ${ }^{1}$ Even though Africa accounts for $90 \%$ of this mortality burden for malaria, South-east Asia still suffers considerable mortality and morbidity. Malaria is a major public health problem in Bangladesh. Of the 11 countries of the World Health Organization South East Asian Regional Office, ten countries including Bangladesh are malaria endemic. ${ }^{2,3}$ The majority of the malaria cases occur in the thirteen districts, out of the 67 administrative districts of Bangladesh and are recognized as malaria endemic. Ninety eight percent 
of the malaria case reports come from these thirteen districts. Three out of these thirteen districts, Bandarban, Khagrachari and Rangamati, collectively known as the Chittagong Hill Tracts (CHT) districts, report the highest incidence of malaria within the country. ${ }^{4}$ Severe malaria cases from these districts are usually referred to Chittagong Medical College Hospital $(\mathrm{CMCH})$, the $2^{\text {nd }}$ largest tertiary level hospital in Bangladesh. In 2006 total 538 malaria patient was admitted in pediatrics ward in $\mathrm{CMCH}$ in a single malaria session. Out of them $90 \%$ cases were severe malaria either CM or non-cerebral severe malaria. ${ }^{5}$

In malaria-endemic areas, asymptomatic malaria parasitemia is common. Individuals with coma of a non-malarial cause may happen to be parasitemic and their illness may be wrongly identified as cerebral malaria (CM). ${ }^{6}$ This difficulty is further enhanced by the considerable overlap that exist between the clinical signs of severe malaria and other serious infectious diseases such as pneumonia ${ }^{7}$, and other causes of coma ${ }^{7-9}$, leading to a failure to treat some of these life-threatening diseases. ${ }^{6}$ The need to improve on the diagnosis of severe malaria has lead to the search for more reliable tools for establishing a diagnosis in order to improve on the appropriate and timely use of interventions aimed at reducing the persistently high mortality of severe malaria. ${ }^{10}$

Erythrocytes parasitized by P. falciparum cytoadhere to vascular endothelium and each other, sequestering in the microvasculature of the brain, retina, and other organs. This crucial pathophysiological change of the disease is difficult to assess in the living patient. An exception is the directly accessible retinal microvasculature, which is very similar to the cerebral microvasculature. ${ }^{11}$ Retinal changes observed in patients with severe malaria can, thus, mirror changes in the brain and be of pathophysiological, prognostic, and diagnostic significance. The occurrence of retinal hemorrhages in severe malaria has been known for over 130 years, ${ }^{12}$ but the unique spectrum of retinal signs in African children with cerebral malaria was first described in Malawi in 1993. ${ }^{13}$ This spectrum consists of four components: retinal whitening, vessel discoloration, retinal hemorrhages, and papilloedema. ${ }^{14}$ Vessel discoloration and the pattern of retinal whitening are unique to and retinal hemorrhages are relatively specific for severe malaria, and they can be used for diagnostic purposes in high transmission areas in
sub-Saharan Africa, where it is difficult to distinguish severe malaria from other causes of severe febrile illness presenting with incidental peripheral blood parasitaemia. ${ }^{14}$ Severity of malarial retinopathy in children also has important prognostic significance for both prolonged coma and death. ${ }^{15}$ There is scarcity of study of ocular findings in Bangladeshi severe malaria children. Improvement in the diagnosis of severe malaria, especially $\mathrm{CM}$, and its differentiation from other causes of childhood coma might contribute to improvement case management and possibly a reduction in mortality. So this study was carried out to see the retinal changes in children with severe malaria by using direct and indirect ophthalmoscopy and to see the retinal findings correlate with disease severity.

\section{Methods}

Study site and patients: This prospective observational study was conducted at Department of Pediatrics of CMCH from February 2008 to January 2009 after obtaining approval from ethical committee of Chittagong Medical College. Consecutive patients age between 6 months to 12 years with peripheral blood slide- or rapid diagnostic test (Paracheck) confirmed Plasmodium falciparum malaria were recruited if written informed consent was obtained from the parents or an attending relative. These patients were divided into the two groups: cerebral malaria (Blantyre coma score $\leq 2$ and no improvement following correction of hypoglycemia, within 30 minutes of cessation of seizure activity or within 2 hours of admission $)^{6}$ and severe non-cerebral malaria (a Blantyre coma score $\geq 2$ and any signs of severe malaria based on WHO criteria). Exclusion criteria were patients unable or unwilling to cooperate with eye examination, contraindications to tropicamide and phenylephrine eye drops (angle closure glaucoma or documented allergy), severe corneal scarring or cataracts in both eyes precluding ophthalmoscopy, intracranial space occupying lesion(s), epilepsy, head injury, and chronic renal failure.

Study procedure: On admission, a full history and examination were carried out. Blood samples were obtained for hemoglobin, hematocrit, parasitemia, platelet count, leukocyte count, serum glucose, and full biochemistry. Eye examination included pupillary reaction to light and direct and indirect ophthalmoscopy. Indirect ophthalmoscopy was 
performed after direct ophthalmoscopy to minimize bias. Observations were made by two independent observers who underwent 2 weeks of intensive training to supplement their limited prior experience of direct and indirect ophthalmoscopy. In case of nonmatching findings, the patient was reexamined, and findings were scored by consensus between the observers. Ophthalmoscopy was performed on admission, and in those who had changes on day 1 , it was repeated on next day and at discharge. Two drops of tropicamide $0.5 \%$ or $1 \%$ eye drops, with the addition of $2.5 \%$ phenylephrine, were used for mydriasis.

Drugs and supportive treatments: Patients were treated with Antimalarial medications and supportive treatments in accordance with the 2006 World Health Organization (WHO) guidelines ${ }^{16}$ and hospital guidelines. They were treated with Intravenous (i.v) quinine sulphate $(20 \mathrm{mg} / \mathrm{Kg}$ bolus dose followed by $10 \mathrm{mg} / \mathrm{kg}$ body weight, 8-hourly), i.v quinine was changed to tablet quinine at the same dosage when patient regain consciousness or after 72 hours (whichever was earlier) via nasogastric tube to complete a 7- day course. Patients with severe anemia or respiratory distress were given humidified oxygen and children with severe anemia were transfused with blood. Hypoglycemia was corrected with i.v 10\% dextrose solution. Paracetamol and anticonvulsant therapy were administered as clinically indicated at standard dosage.

Statistical analysis: Statistical analysis was carried out using the SPSS version 12.0. Between group comparison for continuous data was done with independent sample $t$ test and for categorical data with Chi-square or Fisher's Exact test. All the tests were two-sided, and $\mathrm{P}<0.05$ was considered to be statistically significant. Diagnostic accuracy of direct ophthalmoscopy was calculated considering the indirect ophthalmoscopy as gold standard.

\section{Results:}

A total of 130 children were studied, of whom 80 had cerebral malaria and 50 children had non-cerebral severe malaria. Mean age was $5.64 \pm 3.49$ years with almost equal male female distribution (Table 1).

Common presenting severity features in CM group were convulsion and low BCS at admission and in non-cerebral severe malaria group were clinical jaundice and severe anaemia. Other presenting features were oliguria, acidosis and shock (Table-II)

Table I

Baseline features of the enrolled patients with severe malaria

\begin{tabular}{|c|c|c|c|c|}
\hline Variables & & $\begin{array}{l}\text { Cerebral } \\
\text { malaria }\end{array}$ & $\begin{array}{c}\text { Non-cerebral } \\
\text { severe malaria }\end{array}$ & Total \\
\hline Number of the patients, $\mathrm{n}(\%)$ & $80(61.54 \%)$ & $50(38.46 \%)$ & $130(100 \%)$ & \\
\hline Age & $\begin{array}{l}\text { Mean (years) } \\
\pm S D\end{array}$ & $\begin{array}{c}5.69 \\
\pm 3.60\end{array}$ & $\begin{array}{c}5.57 \\
\pm 3.32\end{array}$ & $\begin{array}{c}5.64 \\
\pm 3.49\end{array}$ \\
\hline Sex, n (\%) & $\begin{array}{l}\text { Male } \\
\text { Female }\end{array}$ & $\begin{array}{l}39(48.8 \%) \\
41(51.2 \%)\end{array}$ & $\begin{array}{l}25(50.0 \%) \\
25(50.0 \%)\end{array}$ & $\begin{array}{l}64(49.23 \%) \\
66(50.77 \%)\end{array}$ \\
\hline
\end{tabular}

Data represent No.(\%) of patients or Mean \pm SD; SD: Standard deviation

Table-II

Distribution of clinical features of the enrolled patients

\begin{tabular}{lccc}
\hline Variables & $\begin{array}{c}\text { Cerebral malaria } \\
(\mathrm{n}=80\end{array}$ & $\begin{array}{c}\text { Non-cerebral } \\
\text { severe malaria }(\mathrm{n}=50)\end{array}$ & $\begin{array}{c}\text { Total } \\
(\mathrm{n}=130)\end{array}$ \\
\hline H/O convulsion & $43(53.8 \%)$ & $5(10.0 \%)$ & $48(36.92 \%)$ \\
BCS<2 & $80(100 \%)$ & $0(0 \%)$ & $80(61.54 \%)$ \\
Severe anaemia $(\mathrm{Hb} \%<5 \mathrm{mg} / \mathrm{dl})$ & $7(8.8 \%)$ & $17(34.0 \%)$ & $24(18.46 \%)$ \\
Oliguria/ARF & $27(33.8 \%)$ & $10(20.0 \%)$ & $37(28.46 \%)$ \\
Clinical jaundice & $36(45.0 \%)$ & $30(60.0 \%)$ & $66(50.77 \%)$ \\
Acidosis & $7(8.8 \%)$ & $4(8.0 \%)$ & $11(8.46 \%)$ \\
Shock & $7(8.8 \%)$ & $4(8.0 \%)$ & $11(8.46 \%)$ \\
\hline
\end{tabular}

Data represent No.(\%) of patients 


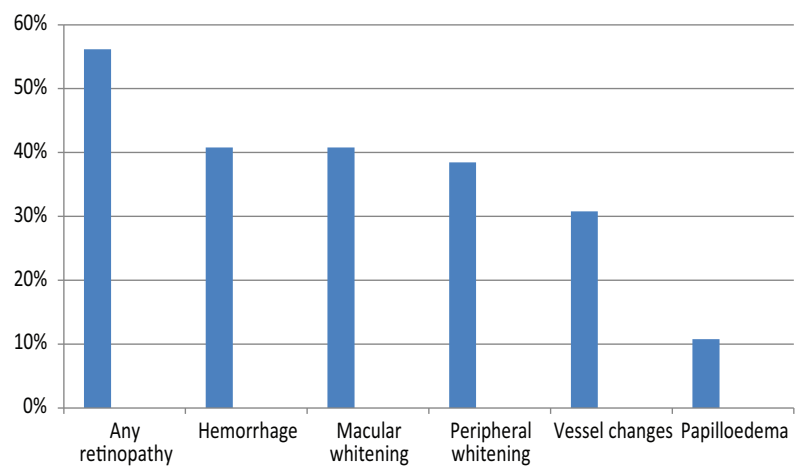

Fig.-1: Retinal changes in patients with severe Plasmodium falciparum malaria by indirect ophthalmoscopy $(n=130)$

Retinal changes in enrolled patients: Features compatible with malarial retinopathy were found in 49 of 80 patients $(61.2 \%)$ with $\mathrm{CM}$ and 24 of 50 patients (48\%) with non cerebral severe malaria. The prevalence of individual features of retinopathy, as seen by indirect ophthalmoscopy, is shown in Figure 1. A comparison of retinal changes by indirect ophthalmosopy between $\mathrm{CM}$ and non-cerebral severe malaria group is shown in Table 3 and it revealed that there was no significant difference between cerebral and non cerebral severe malaria cases.

A comparison of findings by direct and indirect ophthalmoscopy is shown in Table 4 . There was no significant difference in the overall prevalence of retinopathy as assessed by these two techniques, but peripheral abnormalities were better detected by indirect ophthalmoscopy. With indirect ophthalmoscopy as reference, direct ophthalmoscopy had a high sensitivity to detect retinal hemorrhages and papilloedema but was less sensitive to detect retinal whitening.

Table -III

Distribution of retinal changes in cerebral and non-cerebral severe malaria on admission by indirect ophthalmoscopy

\begin{tabular}{lcccc}
\hline Findings & $\begin{array}{c}\text { Cerebral } \\
\text { malaria } \\
(\mathrm{n}=80)\end{array}$ & $\begin{array}{c}\text { Non-cerebral } \\
\text { severe malaria } \\
(\mathrm{n}=50)\end{array}$ & $\begin{array}{c}\text { Total } \\
(\mathrm{n}=130)\end{array}$ & P value \\
\hline Any retinopathy & $49(61.2 \%)$ & $24(48 \%)$ & $73(56 \%)$ & $0.139^{*}$ \\
Retinal hemorrhage & $37(46.2 \%)$ & $16(32 \%)$ & $53(40.77 \%)$ & $0.108^{*}$ \\
Macular whitening & $37(46.2 \%)$ & $16(32 \%)$ & $53(40.77 \%)$ & $0.108^{*}$ \\
Peripheral whitening & $33(41.2)$ & $17(34 \%)$ & $50(38.46 \%)$ & $0.334^{*}$ \\
Vessels change & $27(33.8 \%)$ & $13(26 \%)$ & $40(30.77 \%)$ & $0.352^{*}$ \\
Papilloedema & $11(13.8 \%)$ & $03(6 \%)$ & $14(10.78 \%)$ & $0.116^{*}$ \\
\hline
\end{tabular}

${ }^{*} \mathrm{P}$ value derived from Chi-square test and not significant

Table-IV

Comparison of findings of retinal changes among enrolled patients by direct and indirect ophthalmoscopy $(n=130)$

\begin{tabular}{lcccccc}
\hline \multicolumn{6}{c}{ Retinal changes in severe malarial children } \\
\cline { 2 - 6 } & $\begin{array}{c}\text { Any } \\
\text { retinopathy }\end{array}$ & $\begin{array}{c}\text { Retinal } \\
\text { hemorrhage }\end{array}$ & $\begin{array}{c}\text { Macular } \\
\text { whitening }\end{array}$ & $\begin{array}{c}\text { Peripheral } \\
\text { whitening }\end{array}$ & $\begin{array}{c}\text { Vessel } \\
\text { changes }\end{array}$ & Papilloedema \\
\hline Indirect & $74(56.92 \%)$ & $53(40.77 \%)$ & $53(40.77 \%)$ & $50(38.46 \%)$ & $40(30.77 \%)$ & $14(10.78 \%)$ \\
Direct & $72(55.38 \%)$ & $32(24.62 \%)$ & $54(41.54 \%)$ & $12(9.23 \%)$ & $7(5.38 \%)$ & $14(10.77 \%)$ \\
Sensitivity & $97.30 \%$ & $88.68 \%$ & $100 \%$ & $24.0 \%$ & $17.5 \%$ & $100 \%$ \\
specificity & $100 \%$ & $100 \%$ & $98.7 \%$ & $100 \%$ & $100 \%$ & $100 \%$ \\
\hline
\end{tabular}

Sensitivities and specificities are for direct ophthalmoscopy, using indirect ophthalmoscopy as the reference test 
Table V

Association between retinopathy and survival of patients of severe malaria

\begin{tabular}{|c|c|c|c|c|c|}
\hline \multirow[t]{2}{*}{ Retinopathy } & \multicolumn{2}{|c|}{ Outcome } & \multirow[t]{2}{*}{ Total } & \multirow{2}{*}{$\begin{array}{c}\text { Odds ratio } \\
(95 \% \mathrm{Cl} \text { for OR) }\end{array}$} & \multirow{2}{*}{$\begin{array}{c}\mathrm{P} \\
\text { value }\end{array}$} \\
\hline & Survived & Dead & & & \\
\hline Present & $67(91.8 \%)$ & $06(8.2 \%)$ & 73 (56.2\%) & $1.09(1.017-1.167)$ & $0.035^{\star}$ \\
\hline Absent & $57(100 \%)$ & $0(0 \%)$ & $57(43.8 \%)$ & & \\
\hline Total & $126(96.9 \%)$ & $04(3.1 \%)$ & $130(100 \%)$ & & \\
\hline
\end{tabular}

Data represent No.(\%) of patients ; OR: Odds ratio; Cl: Confidence interval; * $\mathrm{P}$ value derived from Fischer exact test and significant

Out of 130 cases of severe malaria, $6(4.62 \%)$ patients died and all of them had retinopathy. No patients without retinopathy had fatal outcome (Table 5). Duration of hospital stay was significantly higher $(p<0.001)$ among the child with retinopathy than the child without $\{6.32( \pm 1.66)$ days versus $4.77( \pm 1.57)$ days\}. Coma recovery time was also significantly higher among the child with retinopathy (not shown in the result).

The mean duration of hospital stay for the enrolled patients with severe malaria was $5.56( \pm 1.72)$ days. 73 patients with retinal changes on admission were reexamined on $2^{\text {nd }}$ day and at discharge. By the time of discharge, malarial retinopathy had fully cleared in 30 of 73 patients (41.1\%), had improved in 6 of 73 patients $(8.22 \%)$, and was the same as on admission in 37 of 73 patients $(50.68 \%)$. Among the retinal changes papilloedema resolved completely in all patients by the time of discharge and retinal hemorrhage was the most persistent change (Figure 2).

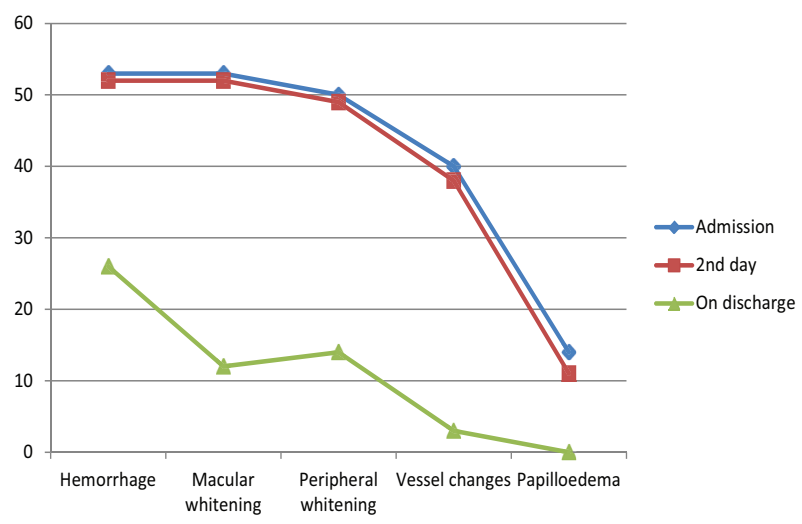

Fig.-2: Retinal changes over time (From admission to discharge from hospital)

\section{Discussion:}

Malarial retinopathy was extensively studied in Africa but received less attention in Bangladesh. To our knowledge, the current study was the first study carried out to evaluate the retinal changes in Bangladeshi child with severe falciparum malaria. This study showed that more than half $(56 \%)$ of pediatric patients with severe malaria have malarial retinopathy on admission when assessed by nonexpert direct and indirect ophthalmoscopy. Retinal hemorrhages are the most easily visible manifestation of malaria in the retina and as such, have the greatest potential as a bedside diagnostic and prognostic sign. This has been proposed for the diagnosis of pediatric cerebral malaria in high transmission settings in sub-Saharan Africa. ${ }^{14}$ In these settings, a positive peripheral blood slide for malaria does not necessarily imply that a child has clinical malaria because of the high background slide positivity rates. In low transmission settings like Bangladesh, the clinical diagnosis of severe malaria is not confounded by asymptomatic peripheral blood parasitemia, and the contribution of ophthalmoscopy to diagnosis is more limited. ${ }^{10}$

Out of 130 patients of severe malaria, 80 were cerebral malaria while 50 were non cerebral malaria and mean $( \pm S D)$ age of them was $5.69( \pm 3.60)$ years and $5.57( \pm 3.32)$ years respectively. There was almost equal representation from both sex (male to female ratio was $1: 1.03)$. These demographic features were in agreement with other similar studies carried out in different countries. ${ }^{14,} 15$

In the present study $61.2 \%$ of child with cerebral malaria had retinopathy and $48 \%$ of non cerebral severe malaria child had retinopathy. Overall retinopathy was present in $56.2 \%$ among severe malaria cases. The present findings match 
prevalence rates found in previous studies in adults with malaria using ophthalmoscopy as a bedside tool. In a study by Kochar and others 17 using a combination of direct and indirect ophthalmoscopy, retinopathy was present in $34 \%$ of patients with cerebral malaria, $24 \%$ of patients with severe noncerebral malaria, and $1 \%$ of patients with uncomplicated falciparum malaria. Studies by Looareesuwan and others ${ }^{18}$ and Davis and others 19 using direct ophthalmoscopy reported the prevalence of retinopathy in patients with cerebral malaria as $14.6 \%$ and $28 \%$, respectively, predominantly as retinal hemorrhages. These discrepancies suggest that the sensitivity of nonexpert direct or indirect ophthalmoscopy for detecting malarial retinopathy is likely to be around $50 \%$.

The current study compared bedside direct and indirect ophthalmoscopy for the assessment of malaria retinopathy. There was little advantage gained from indirect ophthalmoscopy by a non-expert compared with direct ophthalmoscopy regarding overall sensitivity to detect malarial retinopathy except in detecting macular whitening and vessel changes. Macular whitening was found in $41 \%$ in total cases by indirect ophthalmoscopy and only $9.2 \%$ by direct ophthalmoscopy. Vessel changes were found in $30.8 \%$ patient by indirect and $4.5 \%$ by direct ophthalmoscopy. These findings are highly specific for malarial retinopathy and commonly observed in adult patients. ${ }^{15}$ However, in pediatric patients retinal hemorrhages was predominantly described. ${ }^{14}$ Retinal whitening frequently occurs without hemorrhages and can be difficult to see, particularly when it is in the peripheral retina. ${ }^{20}$ In our study sensitivity to detect peripheral whitening and vessel changes by direct ophthalmoscopy were $24 \%$ and $17.5 \%$ respectively. Despite this shortcoming, the present study confirms the utility of assessment by direct ophthalmoscope of malarial retinopathy, especially retinal hemorrhages, as a simple bedside indicator of prognosis in this patient group. Because expert ophthalmological examination of severe malaria in the field setting is unlikely, the results in this study are a realistic reflection of what can be achieved in a real world setting.

The severity of retinal findings was also analyzed in relation to outcome among the cases. All the fatal cases had retinopathy on admission and it was also found that mean duration of hospital stay, parasite counts and coma recovery time have got significance relationship with retinopathy. Sequel of retinal changes on discharge and retinopathy among cases were found highly significance. Longitudinal findings of the fundus showed that papilloedema is very transient. When the patients improved papilloedema resolved rapidly. Retinal hemorrhages were also resolved but gradually. Beare et al., followed-up the patients for 4 weeks after hospital discharge. All were observed to be improving without any secondary retinal sequel. ${ }^{15}$

Limitations of this study include its dependence on the skill of the examining doctor, because ophthalmoscopy is operator dependent and thus, subjective. Study subjects were examined by nonophthalmologists with relatively limited training in ophthalmoscopy. However, this should provide a realistic picture of what might be achieved by similarly skilled non-specialists at the bedside. Another limitation of the current study was that it included neither healthy controls nor patients with uncomplicated malaria. This limited our ability to assess the speciûcity of ûndings for severe malaria, and it was also not possible to determine the background rate of retinal lesions in the population. Moreover, sample size was relatively small and subjects were followed up only for a short in hospital period.

In conclusion, retinal features are an integral part of the clinical picture and ophthalmic observation can contribute to continuing studies of pathophysiological process and therapeutic interventions. The knowledge about malarial retinopathy could improved care of critically ill patient with parasitemia in disease endemic areas and also improve the power of studies to assess interventions aimed to reducing the persistently high mortality of severe malaria. Detection of malarial retinopathy is potentially a low cost, bedside, diagnostic method, which could improve the precision of the clinical diagnosis of severe malaria. Dilated fundoscopy should be the part of the clinical examination and assessment of any patient suspected of having severe malaria.

\section{Acknowledgments}

It was a self funded study. The authors would like to thank all survey participants for their involvement, as well as the Physicians of the respective hospital for their collaboration and cooperation. 


\section{References:}

1. Lopez AD, Mathers CD, Ezzati M, Jamison DT, Murray CJ. Global and regional burden of disease and risk factors, 2001: systematic analysis of population health data. Lancet 2006; 367:1747-1757.

2. Marsh K, Forster D, Warulu C, Mwangl I, Winstanley M, Maesh V, Newton C, Winstanly P, Warn P, Peshu N, Pasvol G, snow R. Indicators of life threatening malaria in African children. N Engl J Med 1995;332:1399-1404.

3. World Health Organization: Severe falciparum malaria. Trans R Soc Trop Med Hyg 2000:9451S90.

4. Bangali AM, Mahmood AH, Rahman M. The malaria situation in Bangladesh. Mekong Malaria Forum 2000;6: 16-23.

5. MIS, Chittagong Medical College Hospital, Chittagong, Bangladesh.

6. White VA, Lewallen S, Beare NA, Molyneux ME, Taylor TE. Retinal pathology of pediatric cerebral malaria in Malawi. PloS ONE. 2009. 4(1): e4317

7. Taylor TE, Fu WJ, Carr Ra, Whitten RO, Mueller JS, Fosiko NG, Lewallen S,Liomba NG, Molyneux ME: Differentiating the pathologies of cerebral malaria by postmortem parasite counts. Nat Med 2004;10: 143-145.

8. Kallander K, Nsungua-Sabati J, Peterson s: symptom overlap for malaria and pneumoniapolicy implications for home management stratagies. Acta trop 2004;90:211-214.

9. English M, punt J, Mwangi I, McHungh K, Marsh k. Clinical overlap between malaria and severe pneumonia in African children in hospital. trans R Sac Trop Med Hyg 1996;90:658-662

10. Dyffy P, Fried M. Malaria: new diagnosis for an old problem. Am J Trop Med Hyg 2005;73:482483.
11. Beare NA, Glover SJ, Molyneux M. Images in Clinical Tropical Medicine Malarial Retinopathy in Cerebral Malaria. Am. J. Trop. Med. Hyg 2009; 80(2): 171.

12. Poncet F. De la retino-choroidite palustre . Ann Ocul (Paris) 1878;79: 201-218 .

13. Lewallen S, Taylor TE, Molyneux ME, Wills BA, Courtright $P$. Ocular fundus findings in Malawian children with cerebral malaria. Ophthalmology 1993;100:857-861.

14. Beare NA, Taylor TE, Harding SP, Lewallen S, Molyneux ME. Malarial retinopathy: a newly established diagnostic sign in severe malaria. Am J Trop Med Hyg 2006;75:790-797

15. Beare NA, Southern C, Chalira C, Taylor TE, Molyneux ME, Harding SP. Prognostic significance and course of retinopathy in children with severe malaria . Arch Ophthalmol 2004;122:1141-1147.

16. World Health Organization. Guidelines for the Treatment of Malaria.2006. Available at: http:// www.who.int/malaria/docs/Treatment Guidelines 2006.

17. Kochar DK, Shubha K, Kumawat BL, Thanvi I, Joshi A, Vyas SP. Ophthalmoscopic abnormalities in adults with falciparum malaria. QJM 1998; 91: 845-852.

18. Looareesuwan S, Warrell DA, White NJ, Chanthavanich $\mathrm{P}$, Warrell MJ, Chantaratherakitti S, Changswek S, Chongmankongcheep L, Kanchanaranya C. Retinal hemorrhage, a common sign of prognostic significance in cerebral malaria. Am J Trop Med Hyg 1993; 32:911-915.

19. Davis MW, Vaterlaws AL, Simes J, Torzillo P. Retinopathy in malaria. P N G Med J1982; 25:19-22.

20. Maude RJ, Hassan MU, Beare NA. Severe retinal whitening in an adult with cerebral malaria. Am J Trop Med Hyg 2009;80: 881. 